\title{
Identifying the Riemann zeros by periodically driving a single qubit
}

\author{
Ran He $\odot,{ }^{1,2}$ Ming-Zhong Ai, ${ }^{1,2}$ Jin-Ming Cui $\odot,{ }^{1,2, *}$ Yun-Feng Huang, ${ }^{1,2, \dagger}$ Yong-Jian Han $\odot,{ }^{1,2, \ddagger}$ Chuan-Feng Li $\odot,{ }^{1,2, \S}$ \\ Tao Tu, ${ }^{1,2}$ C. E. Creffield ${ }^{3},{ }^{3}$ G. Sierra, ${ }^{4}$ and Guang-Can Guo ${ }^{1,2}$ \\ ${ }^{1}$ CAS Key Laboratory of Quantum Information, University of Science and Technology of China, Hefei 230026, People's Republic of China \\ ${ }^{2}$ CAS Center For Excellence in Quantum Information and Quantum Physics, University of Science and Technology of China, \\ Hefei 230026, People's Republic of China \\ ${ }^{3}$ Departamento de Física de Materiales, Universidad Complutense de Madrid, 28040 Madrid, Spain \\ ${ }^{4}$ Instituto de Física Teórica, UAM, CSIC, 28049 Madrid, Spain
}

(Received 19 March 2019; accepted 3 March 2020; published 9 April 2020)

\begin{abstract}
The Riemann hypothesis, one of the most important open problems in pure mathematics, implies the most profound secret of prime numbers. One of the most interesting approaches to solving this hypothesis is to connect the problem with the spectrum of the physical Hamiltonian of a quantum system. However, none of the proposed quantum Hamiltonians has been experimentally feasible. Here we report an experiment using a Floquet method to identify the first nontrivial zero of the Riemann $\zeta$ function and the first two zeros of Pólya's function. Through properly designed periodically driving functions, the zeros of these functions are characterized by the occurrence of crossings of quasienergies when the dynamics of the system is frozen. The experimentally obtained zeros are in good agreement with their exact values. Our study provides the experimental realization of the Riemann zeros in a quantum system, which may provide insights into the connection between the Riemann function and quantum physics.
\end{abstract}

DOI: 10.1103/PhysRevA.101.043402

\section{INTRODUCTION}

The Riemann zeta function is the analytical extension of the Dirichlet series, defined for $s>1$,

$$
\zeta(s):=\sum_{n=1}^{\infty} \frac{1}{n^{s}},
$$

to the complex plane. The Riemann hypothesis (RH) [1] states that all nontrivial zeros of the Riemann $\zeta$ function lie on the critical line $\operatorname{Re}[s]=\frac{1}{2}$, i.e., all nontrivial zeros have the form $s=\frac{1}{2}+i E$, where $E$ are real numbers. The $\mathrm{RH}$ is one of the most important unsolved problems in the field of pure mathematics because it is closely connected to many fundamental mathematical problems [2,3] such as the distribution of prime numbers [4].

At a numerical level, more than $10^{13}$ zeros of the Riemann $\zeta$ function have been identified using a classical computer [5]. However, a complete proof of the RH still needs to explored. At an experimental level, an electromechanical analog device [6] has been used to investigate the Riemann $\zeta$ function in the critical strip. Several other physical implementations have been proposed in both classical systems, such as by the angular nodes separating the side lobes of a far-field diffraction pattern, e.g., in optics or acoustics [7,8], and quantum systems, such as by two entangled quantum systems

\footnotetext{
*jmcui@ustc.edu.cn

†hyf@ustc.edu.cn

${ }^{\ddagger}$ smhan@ustc.edu.cn

§cfli@ustc.edu.cn
}

[9]. Among these approaches, the Pólya-Hilbert conjecture (PHC), which suggests that the imaginary part $E$ in the Riemann zeros corresponds to the eigenvalues of a quantummechanical Hamiltonian, is a fascinating one. In this case all the Riemann zeros will be of the form $\frac{1}{2}+i E$ with $E$ real, which is the statement of the RH. This conjecture was first proposed by Pólya and Hilbert based on the fact that all eigenvalues of a physical Hamiltonian are real $[10,11]$. The PHC is also known as the spectral approach to the RH. Because of the unexpected association between the RH and quantum physics, and particularly the supporting evidence from random matrix theory [12-14] and quantum chaos [15,16], several researchers have attempted to construct a suitable quantum Hamiltonian [17-22]. The $x p$ model is a well-known example $[17,23,24]$, however no such Hamiltonian has been implemented in a real quantum system; for example, more recently an operator related to the $x p$ Hamiltonian whose eigenvalues correspond exactly to the Riemann zeros has been found [25], but unfortunately it is not Hermitian and therefore it is not a proper Hamiltonian.

Recently, a very different approach along these lines was proposed in Ref. [26]. In fact, a correspondence was established between the nontrivial zeros of the Riemann $\Xi$ function and the degeneracy of the quasienergies in a periodically driven qubit (rather than the eigenvalues of a static quantum Hamiltonian). This approach extends the PHC to a driven system and sheds light upon the association between the RH and quantum physics. In particular, the periodic-driving approach is experimentally achievable, although it requires demanding experimental conditions such as a long coherence time, low operational error, and low state-preparation and measurement (SPAM) error. In this study we report an 
experiment to precisely identify the first nontrivial zero of the Riemann $\zeta$ function and the first two zeros of Pólya's function utilizing this Floquet approach.

\section{DYNAMICS OF A FLOQUET SYSTEM}

Because of their rich dynamics and flexibility, periodically driven systems have been extensively used to explore different phenomena such as topological insulators $[27,28]$, nonequilibrium dynamics [29], and time crystals [30,31]. Generally, when a system is driven by the periodic Hamiltonian $H(t)=$ $H(t+T)$ (where $T$ is the period), its dynamics can be effectively described by the Floquet formalism [32-34], i.e., the Floquet equation

$$
\left(H(t)-i \hbar \frac{\partial}{\partial t}\right)\left|\psi_{j}(t)\right\rangle=\epsilon_{j}\left|\psi_{j}(t)\right\rangle
$$

where the state $\left|\psi_{j}(t)\right\rangle$ is a Floquet state satisfying the periodic condition $\left|\psi_{j}(t)\right\rangle=\left|\psi_{j}(t+T)\right\rangle$ and $\epsilon_{j}$ is the quasienergy corresponding to the Floquet state $\left|\psi_{j}(t)\right\rangle$. The Floquet state and the quasienergy are analogs of the eigenstate and the eigenvalue in the time-independent case. The state of the driven system is the superposition of the Floquet states, i.e.,

$$
|\Psi(t)\rangle=\sum a_{j} \exp \left(-i \epsilon_{j} t\right)\left|\psi_{j}(t)\right\rangle,
$$

where $a_{j}$ is the time-independent coefficient determined by the initial state $\Psi(0)$. The Floquet states reflect the short-time response of the system to the driving field within one period, whereas the quasienergies determine the long-term dynamics $[34,35]$.

We consider the periodic-driving Hamiltonian of a qubit system as

$$
H_{E}(t)=\hbar\left[-J \sigma_{x}+J f_{E}(t) / 2 \sigma_{z}\right],
$$

where $f_{E}(t)$ is the periodic driving field (with period $T$ ), i.e., $f_{E}(t)=f_{E}(t+T), E$ is the driving parameter, and $J$ is the tunneling frequency. Generally, it is difficult to exactly solve the Floquet equation (2); however, in the strong-driving limit, where the frequency $\omega=2 \pi / T$ dominates $J$, e.g., $\omega \gg J$, Eq. (2) can be solved by treating the time-independent part of the Hamiltonian $\left(-J \sigma_{x}\right)$ as a perturbation [36,37]. Based on the first-order expansion of $J$, the quasienergies of the driving qubit are given by $\epsilon_{ \pm}= \pm \hbar\left|J_{\text {eff }}\right|$, where

$$
J_{\mathrm{eff}}=\frac{J}{T} \int_{0}^{T} d t e^{-i F_{E}(t)}
$$

is the effective tunneling between the two Floquet states and

$$
F_{E}(t)=\int_{0}^{t} d t_{1} f_{E}\left(t_{1}\right)
$$

Therefore, we engineer effective tunneling via periodic driving.

\section{PERIODIC-DRIVING FUNCTION FOR THE RIEMANN छ FUNCTION AND PÓLYA'S FUNCTION}

As shown in Sec. VI, if the driving field $f_{E}(t)$ in Eq. (4) is carefully designed, the effective tunneling $\operatorname{Re}\left[J_{\text {eff }}(E)\right]$ can be proportional to the Riemann $\Xi$ function, i.e., $\operatorname{Re}\left[J_{\text {eff }}(E)\right] \propto$ $\Xi(E)$. The Riemann $\Xi$ function, whose zeros coincide with
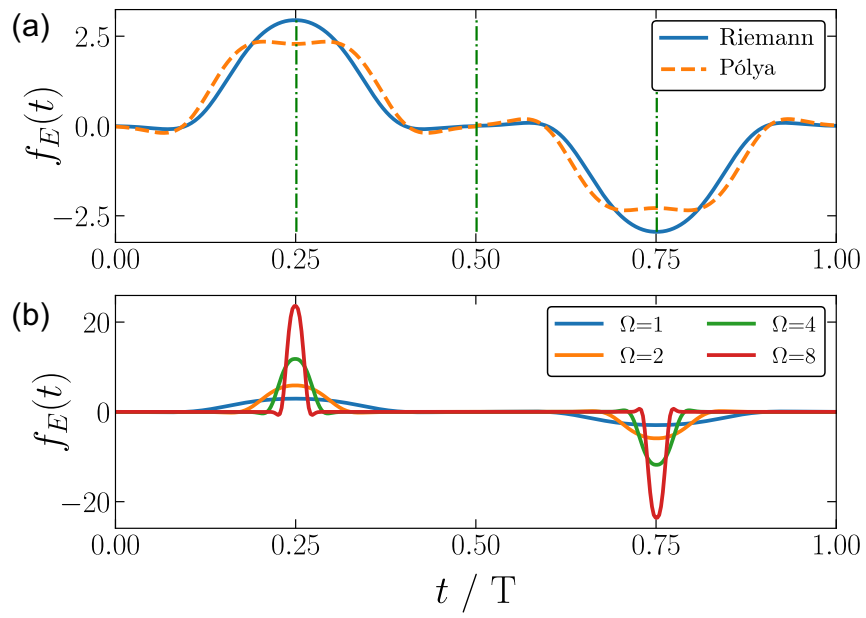

FIG. 1. (a) Driving functions $f_{E}(t)$ for the Riemann $\Xi$ function (blue solid line) and Pólya's function (orange dashed line) for $E=4$. Four copies (separated by green dash-dotted lines) of the truncated $R_{E}(t)$ [Eq. (15)] and $P_{E}(t)$ functions [Eq. (18)] in the interval $0 \leqslant$ $t<\pi / 2$ are joined together to obtain the continuous periodic-driving function $f_{E}(t)$ with a vanishing time average. The driving period is $T$. (b) Pulse shape with different $\Omega$. The period $T$ is fixed at a constant value $2 \pi$. The driving pulse becomes narrower and higher as $\Omega$ increases. Note that a large $\Omega$ would ensure that the system is well within the high-frequency regime. In our experiment, $\Omega$ is set to 8 .

the nontrivial zeros of the Riemann $\zeta(s)$ function, is $\Xi(E)=$ $\frac{1}{2} s(s-1) \Gamma\left(\frac{s}{2}\right) \pi^{-s / 2} \zeta(s)$, where $s=\frac{1}{2}+i E$. Consequently, when $E$ is the zero of $\Xi(E), J_{\text {eff }}(E)$ will vanish, indicating that the dynamics of the system is frozen. This phenomenon is the so-called coherent destruction of tunneling (CDT) $[34,38]$. Therefore, the zeros of the Riemann $\Xi$ function which coincide with the nontrivial zeros of $\zeta(s)$ can be obtained by detecting the degeneracy of the quasienergies as the parameter $E$ varies.

For Riemann function, the driving function is defined as

$$
R_{E}(t)=-\frac{\Phi^{\prime}(t) \cos (E t / 2)-(E / 2) \Phi(t) \sin (E t / 2)}{\sqrt{\Phi^{2}(0)-[\Phi(t) \cos (E t / 2)]^{2}}},
$$

where $\Phi(t)=2 \pi e^{5 t / 4} \sum_{n=1}^{\infty}\left(2 \pi e^{t} n^{2}-3\right) n^{2} e^{-\pi n^{2} e^{t}} \quad$ [for details, see Eq. (15) in Sec. VI]. To achieve the desired effective tunneling, the driving function in one period is formed by four segments: The first segment $(0 \leqslant t \leqslant \pi / 2)$ can be described as $f_{E}(t)=R_{E}(\pi / 2-t)$; the second segment $(\pi / 2 \leqslant t \leqslant \pi)$ is obtained by reflection transformation of the first segment along $t=\pi / 2$, i.e., $f_{E}(t)=f_{E}(\pi-t)$; and the third and fourth segments $(\pi \leqslant t \leqslant 2 \pi)$ can be constructed as $f_{E}(t)=$ $-f_{E}(2 \pi-t)$. The driving function in the whole period is shown in Fig. 1(a). Note that this function is continuous, has a time average of zero, and satisfies the required parity condition (details in Sec. VI).

Similarly, Pólya's function $\Xi^{*}(E)$, which can be viewed as a smoothed version of the Riemann $\Xi(E)$ function, also has an engineered driving function $P_{E}(t)$ [the form of the function is shown in Fig. 1(a) and Eq. (18) in Sec. VI]. By detecting the degeneracy of quasienergies by scanning the parameter $E$ in the driving field, its zeros can be obtained as well. 
To ensure the validity of the previous statement, two contradictory conditions need to be guaranteed: (a) the highfrequency condition in Eq. (4) $(\omega=2 \pi / T \gg J)$, which validates the perturbation expansion in the Floquet formalism, and (b) the large- $T$ condition, which guarantees that the effective tunneling converges well to the desired function whose zeros are going to be located. Actually, since the driving functions $R_{E}(t)$ and $P_{E}(t)$ decay rapidly to zero, truncating the functions at $T_{0}=\pi / 2$ is enough to ensure the convergence requirement. To further satisfy the high-frequency condition, the period of the driving function is fixed to $2 \pi$ and the driving field $f_{E}(t)$ is rescaled as $\Omega f(\Omega t)$, where $\Omega>1$. The rescaled driving field is shown in Fig. 1(b). As $\Omega$ increases, the driving pulses get narrower and higher and the quasienergy spectrum of the system approaches the high-frequency limit. In the experiment, we set $\Omega=8$.

\section{EXPERIMENTAL SETUP}

To demonstrate the previous theories, the ion-trap simulator is an ideal setup. The interacting Hamiltonian of the ion and driving microwave field natively has the form of Eq. (4). Ion traps feature long coherence times and high fidelity of both operation and detection, which are critical to observe the CDT because dozens of driving cycles are necessary. A system with a short coherence time will degrade the achievable precision of the measured zeros. In the experiment, the qubit to be driven is encoded in the 12.6-GHz hyperfine clock transition $|0\rangle \equiv{ }^{2} S_{1 / 2}\left|F=0, m_{F}=0\right\rangle$ and $|1\rangle \equiv{ }^{2} S_{1 / 2} \mid F=$ $\left.1, m_{F}=0\right\rangle$ in a single ${ }^{171} \mathrm{Yb}^{+}$ion confined in an rf trap [39]. This transition is first-order magnetic-field insensitive and a long coherence time of $10 \mathrm{~min}$ has been observed using sympathetic cooling and dynamical decoupling [40].

After $1 \mathrm{~ms}$ of Doppler cooling, the qubit is initialized to the ground state $|0\rangle$ with a probability of $99.9 \%$. Then a modulated microwave-driving pulse $B\left(t^{\prime}\right)=B_{0} \cos \left[\omega_{01} t^{\prime}+J \phi(t=\right.$ $\left.J t^{\prime}\right)$ ] is generated using a programmable arbitrary waveform generator, where $\omega_{01}$ is the qubit transition frequency and $t^{\prime}=t / J$ is the real time in units of $s$ and $\phi(t)=F(t) / 2$. Moving to the rotating frame of the microwave using the unitary transformation $e^{-i\left[\omega_{01} t^{\prime}+J \phi\left(J t^{\prime}\right) / 2\right] \sigma_{z}}$ and applying the rotating-wave approximation, we obtain the microwave and the atom interaction Hamiltonian (4) [41]. Note that the tunneling frequency $J$ is set to $(2 \pi) 4 \mathrm{kHz}$, which corresponds to a Rabi time of $250 \mu \mathrm{s}$. Moreover, the scale parameter $\Omega$ is set to 8 and the qubit is then driven for time $t^{\prime}=m T^{\prime}$, where $m$ is an integer and $T$ is the fundamental driving period $T^{\prime}=2 \pi / J$. Subsequently, a resonant $\frac{\pi}{2}$ microwave pulse is applied to transfer the targeted measurement basis to $|+\rangle=\frac{1}{\sqrt{2}}(|0\rangle+|1\rangle)$. Finally, a $400-\mu$ s pulse of a $369.5-\mathrm{nm}$ laser is immediately used for fluorescence detection. When more than one photon is detected, the measurement result is denoted by 1 ; otherwise, it is denoted by 0 . Many repetitions are performed to obtain the probability. Here we use the probability $P_{|+\rangle}$projected onto the basis $|+\rangle=\frac{1}{\sqrt{2}}(|0\rangle+|1\rangle)$ as the primary indicator of CDT (the details of the selection of the projection basis can be found in Sec. VI). Once we determine that $P_{|+\rangle}$is almost constant at some $E, P_{|0\rangle}$ and $P_{|i\rangle}$ are further measured to confirm the occurrence of CDT, where $|i\rangle=\frac{1}{\sqrt{2}}(|0\rangle+i|1\rangle)$

\section{EXPERIMENTAL RESULTS}

\section{A. Riemann $\boldsymbol{\Xi}(\boldsymbol{E})$ function}

To detect the CDT, we scan the driving parameter $E$ and measure $P_{|+\rangle}\left(T_{m}^{\prime}, E\right)$, which denotes the probability projected on the basis $|+\rangle$ at an integer time interval $T_{m}^{\prime}$. To quantitatively measure the deviation between the measured evolution and the CDT, we define the sum of the values of the residuals (SOR) as $S\left(T_{m}^{\prime}, E\right)=\sum_{m} P_{|+\rangle}\left(T_{m}^{\prime}, E\right)-P_{\mathrm{CDT}}\left(T_{m}^{\prime}\right)$, where $P_{\mathrm{CDT}}\left(T_{m}^{\prime}\right)$ is the probability when CDT occurs. Here $P_{\mathrm{CDT}}\left(T_{m}^{\prime}\right)$ is determined by the prepared state and the measurement basis, e.g., in our current experiment, $P_{\mathrm{CDT}}\left(T_{m}^{\prime}\right)=0.5$. The CDT occurs at a certain $E$ where the measured probabilities $P_{|+\rangle}\left(T_{m}^{\prime}, E\right)$ are constant for all $T_{m}^{\prime}$. Therefore, $S\left(T_{m}^{\prime}, E\right)=0$ is the evidence of the CDT in our experiment. That is to say, $S\left(T_{m}^{\prime}, E\right)$ has the same zeros $E$ with the Riemann $\Xi(E)$ function. A root-finding method such as the bisection method can be used. In the experiment, however, we scan the driving parameter $E$ linearly in order to demonstrate the overall characteristic of our method. Once we find the interval of $E$ in which the SOR changes sign, we decrease the scanning steps to find a smaller interval.

We first detect the zeros of the Riemann $\Xi(E)$ function. One period of the fundamental driving function $f_{E}(t)$ is depicted in Fig. 1. To identify the $E$ corresponding to the CDT, $E$ is first scanned between 10 and 18 with a step size $\Delta E=1$. As can be seen in Fig. 2(a), when $E$ approaches 14, the probability $P_{|+\rangle}$on the basis $|+\rangle$is stable and barely changes from 0.5 . Note that $S(E=14)=-0.036 \pm 0.014$ and $S(E=15)=0.074 \pm 0.014$ have opposite signs, which suggests that there is at least one zero between $E=14$ and 15 , e.g., the first approximation of the Riemann zero $E_{0}$ is the midpoint 14.5 with a maximum possible error of 0.5 , which is half the interval length.

To determine a better value of $E$ at which the CDT may appear, we utilize a smaller $\Delta E$ and scan $E$ from 13.5 to 14.5. The experimental results are depicted in Figs. 2(c) and 2(d). Because $S(E=14.2)=-0.012 \pm 0.009$ is always negative and $S(E=14.4)=0.009 \pm 0.009$ is positive, we infer that the second approximation of the Riemann zero is $E=14.3 \pm$ 0.1 . This is close to the exact first zero of the Riemann $\zeta$ function $\zeta\left(\frac{1}{2}+i E\right)$, i.e., $E=14.1347$. As shown below, when $\Omega$ is higher, the result will be more precise.

The experiment is repeated 40000 times in Fig. 2(a) and 160000 times in Fig. 2(c). The error bars in Figs. 2(a) and 2(c) indicate the statistical error within one standard deviation. The error bars of the SORs in Figs. 2(b) and 2(d) are the sums of the statistical errors of the corresponding $m$ data points $p_{0}(m, E)$ in Figs. 2(a) and 2(c), respectively.

\section{B. Pólya's function $\Xi^{*}(E)$}

We now focus on identifying zeros of Pólya's function. Similar to the Riemann $\Xi$ function situation, we first scan the parameter $E$ with a step size $\Delta E=1$. The experimental results are shown in Figs. 3(a) and 3(b). Because 
(a)

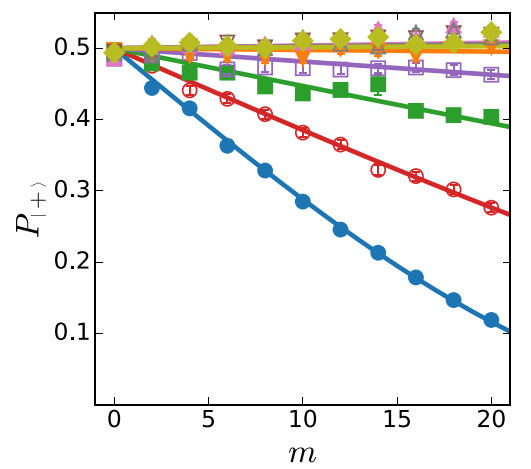

(b)

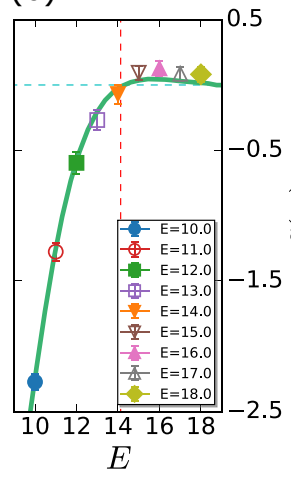

(c)

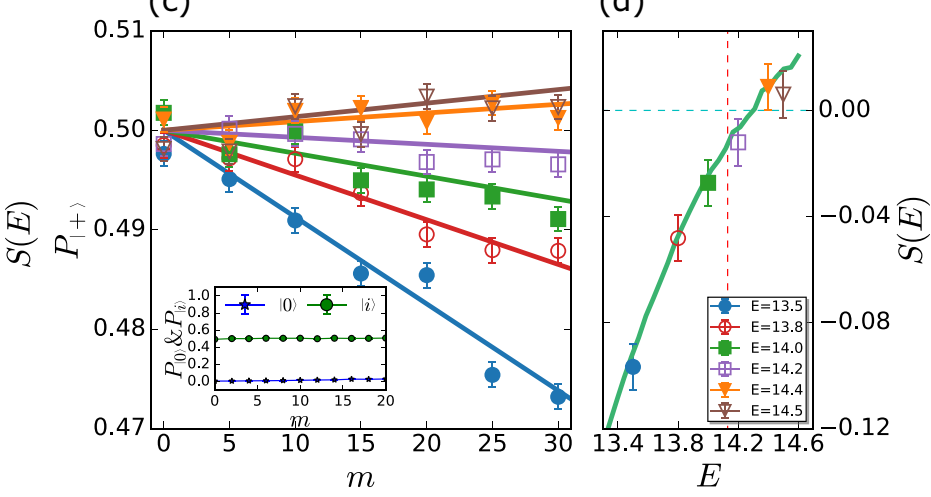

FIG. 2. Dynamics of the probability $P_{|+\rangle}\left(E, t^{\prime}=m T^{\prime}\right)$ with driving parameter $E$ near the first zero of the Riemann $\Xi(E)$ function. (a) In our experiment, the driving comprises up to 20 identical periods of $f_{E}(t)$. With different driving functions (characterized with different parameters $E)$, the probability $P_{|+\rangle}$at time $m T\left(T=2 \pi\right.$ is the period) behaves differently. Under the tailored driving function $f_{E}(t), \operatorname{Re}\left[J_{\text {eff }}(E)\right]$ is proportional to $\Xi(E)$. Once the dynamics is frozen, i.e., CDT occurs, the associated $E$ corresponds to the zero of $\Xi(E)$. It is clear from (a) that the evolution is nearly frozen (flat) when $E$ approaches 14 (CDT). (b) The sums of the values of the residuals (blue horizontal dashed line) $S(E=14)=-0.036 \pm 0.014$ and $S(E=15)=0.074 \pm 0.014$ have opposite signs, which suggests that there is at least one zero between $E=14$ and 15. The green solid curve shows the theoretical values of the SOR. (c) For better precision, we obtain measurements from $E=13.5$ to $E=14.5$ with a smaller step $\Delta E$ and drive the system up to 30 periods. Each measurement is repeated 160000 times. The inset in (c) depicts the measured probabilities $P_{|0\rangle}\left(E, t^{\prime}=m T^{\prime}\right)$ and $P_{|i\rangle}\left(E, t^{\prime}=m T^{\prime}\right)$, where $|i\rangle=(|0\rangle+i|1\rangle)$ with $E=14.0$. The SOR in (d) shows that $S(E=14.2)=-0.012 \pm 0.009$ and $S(E=14.4)=0.009 \pm 0.009$ have opposite signs. Therefore, the CDT happens between $E=14.2$ and $E=14.4$ and one Riemann zero is measured to be $14.3 \pm 0.1$ [the exact first zero of $\Xi(E)$ is 14.1347 , indicated by the red vertical dashed line].

$S(E=9)=-0.062 \pm 0.014$ and $S(E=10)=1.162 \pm 0.013$ have opposite signs, the first zero of $S(E)$ is approximated to be $E=9.5 \pm 0.5$. To obtain a more precise value of $E$, we scan $E$ from 8.5 to 9.5 with a step size of $\Delta E=$ 0.1 ; the results are shown in Figs. 3(c) and 3(d). Because $S(E=9.0)=-0.081 \pm 0.014$ and $S(E=9.1)=0.061 \pm$ 0.014 have opposite signs, the zero of $S(E)$ and $\Xi^{*}(E)$ is measured to be $9.05 \pm 0.05$, which is close to the exact first zero of Pólya's function $\Xi^{*}(E)$, i.e., $E=8.993$. Furthermore, we measured the second zero of Pólya's function, which is $19.5 \pm 0.5$, as shown in Fig. 4. For each point, the experiment was repeated 40000 times.

(a)

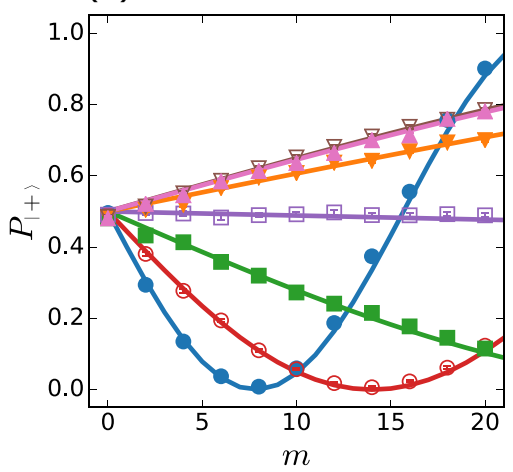

(b)

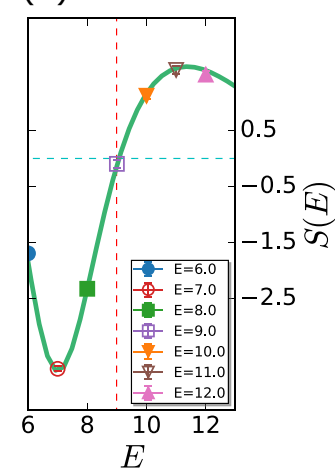

The experimental result and the theoretical prediction in Fig. 3(d) are slightly larger than the exact zero 8.993 because the scale factor $\Omega$ is not large enough to satisfy the high-frequency limit. To demonstrate that the zeros can be better approached with a higher value of $\Omega$, the behaviors of $P_{0}\left(t^{\prime}=20 T^{\prime}, E\right)$ at $E=8.9,9.0$, and 9.1 with varying $\Omega$ are shown in Fig. 5. The smaller $\Omega(\Omega<6)$ introduces a larger deviation from the actual zero of Pólya's function $\Xi^{*}(E)$, e.g., when $\Omega \leqslant 6$, CDT occurs with the driving parameter $E>$ 9.1. Moreover, CDT appears in $9.0<E<9.1$ when $\Omega \geqslant 8$ and approaches the exact value 8.993 as $\Omega$ further increases. Therefore, a higher $\Omega$ is favorable because it helps to obtain

FIG. 3. Dynamics of the probability $P_{|+\rangle}\left(E, t^{\prime}=m T^{\prime}\right)$ with driving parameter $E$ near the first zero of Pólya's function $\Xi^{*}(E)$. (a) The coarse scan of $E$ from 6 to 12 explicitly indicates that the evolution is frozen around $E=9$. (b) The measured (shapes) and theoretical (green solid line) values of the SOR. When $E=9$, the SOR is nearly zero (blue horizontal dashed line). A fine scan around $E=9.0$ with a step of 0.1 is depicted in (c). The inset in (c) depicts the measured probabilities $P_{|0\rangle}\left(E, t^{\prime}=m T^{\prime}\right)$ and $P_{|i\rangle}\left(E, t^{\prime}=m T^{\prime}\right)$ with $E=9.0$. (d) Because $S(E=9.0)=-0.081 \pm 0.014$ and $S(E=9.1)=0.061 \pm 0.014$ have opposite signs, the zero of $S(E)$ and the Pólya's function is measured to be $9.05 \pm 0.05$. The difference from the exact zero of 8.993 (the red vertical dashed line) is because of the limited $\Omega$. 


\section{(a)}

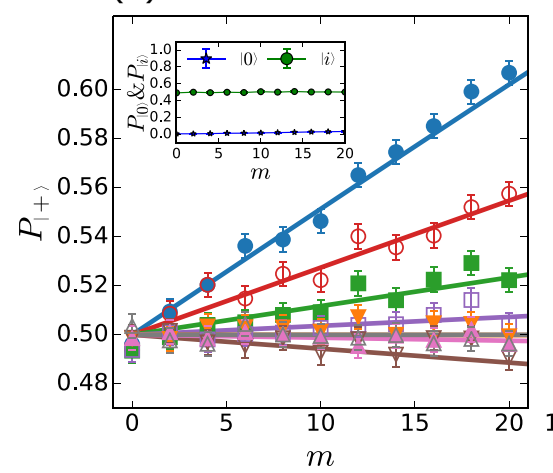

(b)

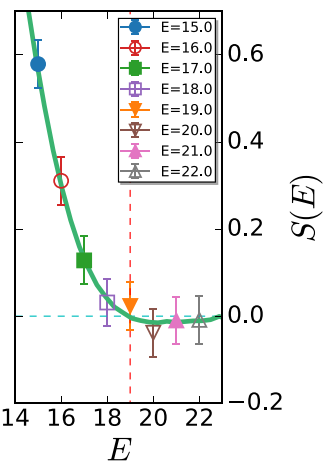

FIG. 4. Dynamics of the probability $P_{|+\rangle}\left(E, t^{\prime}=m T^{\prime}\right)$ with a driving function near the second zero of Pólya's function $\Xi^{*}(E)$. (a) When $E$ increases from 15 to 22, the second CDT and therefore the second zero point of $\Xi^{*}(E)$ appears between $E=19$ and $E=$ 20. The inset in (a) depicts the measured probabilities $P_{|0\rangle}\left(E, t^{\prime}=\right.$ $\left.m T^{\prime}\right)$ and $P_{|i\rangle}\left(E, t^{\prime}=m T^{\prime}\right)$, where $|i\rangle=(|0\rangle+i|1\rangle)$ when $E=19.0$. (b) From the SOR values for each $E$, we can infer that the second zero of Pólya's function is measured to be $E=19.5 \pm 0.5$, which agrees well with the exact zero, i.e., 18.996.

zeros closer to the exact Riemann zeros. However, from an experimental point of view, a larger $\Omega$ results in smaller quasienergies and needs more driving periods to separate different evolution curves with different $E$. This is limited by the coherence time and the stability of the system. Therefore, under the tradeoff, we select $\Omega=8$ in the experiment.

\section{METHODS}

\section{A. Driving function}

We briefly introduce how driving functions can be obtained [26]. On one hand, the effective Hamiltonian of the driving

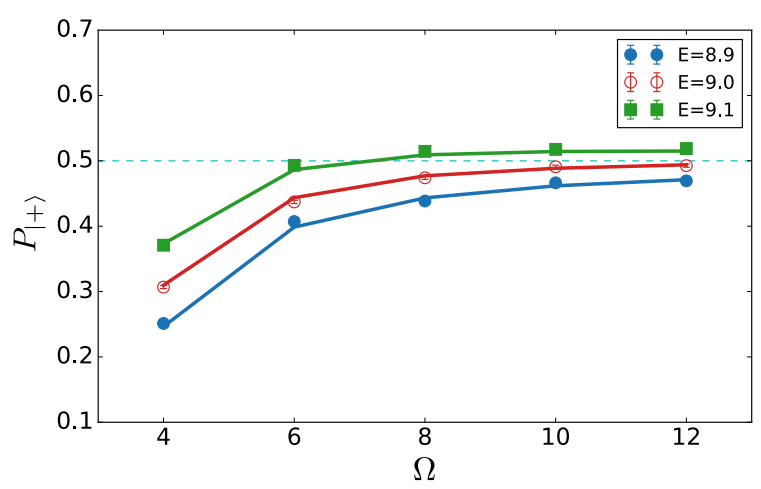

FIG. 5. Dynamics of the probability $P_{|+\rangle}$versus the scale factor $\Omega$ near the first zero of Pólya's function. The larger value of $\Omega$ indicates a better high-frequency approximation and a higher precision of the zeros. The system undergoes CDT with the driving parameter $E>9.1$ when $\Omega=6$; however, when the parameter $\Omega$ increases to 8 , CDT occurs with the parameter $9.0<E<9$. 1 . If $\Omega$ further increases to 12, CDT appears with the parameter $E$ approaching 9.0, which is closer to the exact result of $E=8.993$. Thus, if $\Omega$ keeps increasing, the CDT will theoretically approach the exact zero of the $\Xi^{*}(E)$ function. Moreover, the results show that the limited $\Omega$ is the dominant reason for the deviation in zeros from exact values. system with the Hamiltonian (4) under the first-order perturbation is

$$
H_{\text {eff }}=J_{\text {eff }} \sigma_{x} .
$$

The quasienergies of the driving qubit are given by $\epsilon_{ \pm}=$ $\pm\left|J_{\text {eff }}\right|$, where $J_{\text {eff }}$ is determined by Eq. (5). The real part of $J_{\text {eff }}(E)$ is

$$
\operatorname{Re}\left[J_{\text {eff }}(E)\right]=\int_{0}^{T} d t \cos F_{E}(t)
$$

On the other hand, the Riemann $\Xi(E)$ function can be written as [10]

$$
\Xi(E)=\int_{0}^{\infty} d t \Phi(t) \cos (E t / 2),
$$

where $\Phi(t)=2 \pi e^{5 t / 4} \sum_{n=1}^{\infty}\left(2 \pi e^{t} n^{2}-3\right) n^{2} e^{-\pi n^{2} e^{t}}$ (in this work, we used the first 100 terms). The aim is to find an $F_{E}(t)$ such that the real part of $J_{\text {eff }}(E)$ is proportional to the Riemann $\Xi(E)$ function, i.e.,

$$
\alpha \int_{0}^{\infty} d t \Phi(t) \cos (E t / 2)=\int_{0}^{T} d t \cos F_{E}(t)
$$

where $\alpha$ is the constant of proportionality between $J_{\text {eff }}(E)$ and the $\zeta$ function. For $T$ sufficiently large, we can replace the upper limit of integration on the left to obtain

$$
\alpha \int_{0}^{T} d t \Phi(t) \cos (E t / 2)=\int_{0}^{T} d t \cos F_{E}(t) .
$$

Note that this relation must hold for all sufficiently large values of $T$ (not just one particular value of $T$ ). As a consequence, we can deduce that

$$
F_{E}(t)=\cos ^{-1}[\alpha \Phi(t) \cos (E t / 2)] .
$$

Boundary conditions require $F(t=0)=0$, and thus $\alpha \Phi(0)=1$ and $\alpha=1 / \Phi(0)$. This allows us to write $F(t)$ as

$$
F_{E}(t)=\cos ^{-1}[\Phi(t) / \Phi(0) \cos (E t / 2)] .
$$

Having obtained this result, we can now choose a specific value for $T$. Because $\Phi(t)$ decays rapidly, the integration on the right-hand side can be cut at $T=\pi / 2$. Thus we choose $T=\pi / 2$. By this choice of $F_{E}(t)$ the real part of $J_{\text {eff }}(E)$ becomes proportional to $\Xi(E)$. If $E$ is the zero of the Riemann $\zeta$ function, it will be also the zero of the Riemann $\Xi(E)$ function and the $J_{\text {eff }}(E)$ will vanish.

From Eqs. (6) and (14) the driving function for the Riemann $\Xi(E)$ function can be directly obtained as

$$
R_{E}(t)=-\frac{\Phi^{\prime}(t) \cos (E t / 2)-(E / 2) \Phi(t) \sin (E t / 2)}{\sqrt{\Phi^{2}(0)-[\Phi(t) \cos (E t / 2)]^{2}}},
$$

where $t=J t^{\prime} \in[0, \pi / 2]$ represents a dimensionless parameter [26].

The same method can be used on Pólya's function,

$$
\Xi^{*}(E)=4 \pi^{2}\left[K_{a+i E / 2}(x)+K_{a-i E / 2}(x)\right],
$$

where $x=2 \pi, a=9 / 4$, and $K_{\beta}(t)$ is the modified $K$ Bessel function, $K_{\beta}(x)=\int_{0}^{\infty} d t \cosh (\beta t) e^{-x \cosh t}$. Thus

$$
\Xi^{*}(E)=\int_{0}^{\infty} d t \alpha \cosh (a t) e^{-2 \pi \cosh t} \cos (E t / 2) .
$$


Replacing the upper limit with $\pi / 2$ will only lead to a negligible relative error on the order of $10^{-7}$. From $\operatorname{Re}\left[J_{\text {eff }}(E)\right] \propto$ $\Xi^{*}(E)$ the driving function can be obtained as

$$
P_{E}(t)=-\frac{\phi(t)[a \tanh a t-2 \pi \sinh t-E / 2 \tan (E t / 2)]}{\sqrt{1-\phi(t)^{2}}},
$$

where $a=9 / 4$ and $t=J t^{\prime} \in[0, \pi / 2]$.

Both $R_{E}(t)$ and $P_{E}(t)$ are truncated at $\pi / 2$. The driving function $f_{E}(t)$ constructed by directly repeating $R_{E}(t)$ or $P_{E}(t), m$ times has the following limitations: (i) It is discontinuous; (ii) its average over one period does not vanish, which may heat the cold atom; (iii) the periodic driving field does not have odd parity, which indicates that the quasienergies may form a broad avoided crossing according to the von Neumann-Wigner theorem [42]. To ensure that the driving field $f_{E}(t)$ satisfies the parity requirement $\mathcal{P}: x \rightarrow-x$ and $t \rightarrow t+T / 2$, we joined together four copies of $R_{E}(t)$ or $P_{E}(t)$ as shown in Fig. 1(a) with the total period $T=2 \pi$ (in the experimental setting, the period $T^{\prime}$ is $2 \pi / J$ ). Note that these modified driving functions (introduced in the text) can overcome all of the above problems and remain experimentally achievable.

\section{B. Measurement-basis selection}

Generally, CDT can be detected by projecting the states at time $m T$ onto any basis; however, the sensitivity of the probability against the parameter $E$ is strongly dependent upon the measurement basis. Here we show how to select the measurement basis such that the CDT can be observed in a reasonable driving time and at a higher precision.

In our experiment, the initial state in Eq. (3) is uniformly prepared in $|0\rangle$. We now compare three often-used measurement bases: (i) $|0\rangle$ (basis for $\sigma_{z}$ ), (ii) $|+\rangle$ (basis for $\sigma_{x}$ ), and (iii) $|i\rangle=\frac{1}{\sqrt{2}}\left(|0\rangle+i|1\rangle\right.$ ) (basis for $\sigma_{y}$ ).

(i) If we project the system on the basis $|0\rangle$, the probability at time $t^{\prime}=m T^{\prime}$ with the parameter $E$ will be $P_{|0\rangle}\left(E, t^{\prime}\right)=$ $\frac{1}{2}+\cos \left[2 \epsilon(E) t^{\prime}\right] / 2$, where $\epsilon(E)$ is the quasienergy defined in Eq. (2). For two close parameters $E_{1}$ and $E_{2}$, the difference of their probabilities is $\triangle P_{|0\rangle}=P_{|0\rangle}\left(E_{1}, t^{\prime}\right)-P_{|0\rangle}\left(E_{2}, t^{\prime}\right)=$ $t^{\prime 2}\left[\epsilon^{2}\left(E_{1}\right)-\epsilon^{2}\left(E_{2}\right)\right]+O\left(\left(t^{\prime} \epsilon\right)^{2 k}\right)$, where $O\left(\left(t^{\prime} \epsilon\right)^{2 k}\right)$ is the higher-order even terms.

(ii) If we project the state on the bases $|+\rangle$, the probability is $P_{|+\rangle}=\frac{1}{2}-\sin \left[2 \epsilon(E) t^{\prime}\right] / 2$. Similarly, the difference of the probabilities of two close parameters $E_{1}$ and $E_{2}$ is

$$
\triangle P_{|+\rangle}=t^{\prime}\left[\epsilon\left(E_{1}\right)-\epsilon\left(E_{2}\right)\right]+O\left(\left(t^{\prime} \epsilon\right)^{2 k+1}\right),
$$

where $O\left(\left(t^{\prime} \epsilon\right)^{2 k+1}\right)$ gives the higher-order odd terms.

(iii) If we project the system on the basis $|i\rangle$, the probability $P_{|i\rangle}$ will sinusoidally oscillate near 0.5 , with the amplitude $A$ being much smaller than $1(A \simeq 0.005$ for the Riemann function). The probability difference will be too small to be measured experimentally.

The quantity $t^{\prime} \epsilon$ is much smaller than 1 when $E$ approaches CDT, which indicates $\Delta P_{|0\rangle}, \Delta P_{|i\rangle} \ll \Delta P_{|+\rangle}$. To obtain precise values of zeros of the Riemann $\Xi(E)$ and Pólya functions, we need to separate $P_{|+\rangle}\left(E_{1}, t^{\prime}\right)$ from $P_{|+\rangle}\left(E_{2}, t^{\prime}\right)$ as far as possible, where $E_{1}$ and $E_{2}$ are close to each other. The previous analysis shows that $P_{|+\rangle}$is much more sensitive to the parameter $E$ than $P_{|0\rangle}$ and $P_{|i\rangle}$. Therefore, in our experiment, we select $P_{|+\rangle}$as our primary CDT indicator. Furthermore, Eq. (19) indicates that if we want to have a larger $\Delta P_{|+\rangle}$, a longer driving time $t^{\prime}$ is expected. In the experiment, because of the SPAM error (99.5\% in our system), the best distinguishable $\Delta P_{|+\rangle}$is around 0.005 . If CDT occurs at $E_{2}$ and $\Delta E=$ $0.1, \epsilon\left(E_{1}\right)-\epsilon\left(E_{2}\right)$ will be of the order of $\left(5 \times 10^{-4}\right) / T^{\prime}$, which indicates that the driving time $t$ should be at least $10 T^{\prime}$ according to Eq. (19). Moreover, the coherence time should be longer than 2000 periods ( $\sim 500 \mathrm{~ms}$ in our case). However, to identify the second zero of the Riemann function, $t^{\prime}$ should be longer than $1000 T^{\prime}$ and the coherence time needs to be over $10^{5} T^{\prime}(\sim 30 \mathrm{~s}$ in our case).

\section{OBSERVATION OF CDT IN A TRAPPED-ION QUBIT}

Unlike the original proposal that $J_{\text {eff }}$ can be directly measured by the free-expansion rate of a Gaussian wave packet of cold atoms [26,43,44], we detect the vanishing of the effective tunneling $J_{\text {eff }}$ using the frozen dynamics in our trapped ion. Generally, the system is in the state described by Eq. (3) whose evolution depends only upon the quasienergies. When CDT occurs, the vanishing quasienergies in Eq. (3) merely contribute a global phase to the after-integer periods, i.e., $|\Psi(t+k T)\rangle=e^{i \varphi}|\Psi(t)\rangle$, where $k$ is an integer, $T$ is the period of the driving, and $\varphi$ is the global phase, i.e., the state is frozen at multiple periods. Therefore, when the CDT occurs, the vanishing of the quasienergies can be directly determined by the constant population on every measurement basis after multiple periods. For this experiment, we first detect the $|+\rangle$ basis to identify the frozen point of $E$. Subsequently, we confirm that the CDT indeed occurs by detecting the state evolution onto bases $|0\rangle$ and $|i\rangle$.

\section{CONCLUSION}

In conclusion, we have periodically driven a two-level quantum system and identified that the special values of a control parameter where the CDT phenomenon happens coincides with the first nontrivial zero of the Riemann $\zeta$ function and the first two zeros of Pólya's function. By observation of the vanishing of the quasienergies of a periodically driven system, the Riemann zeros can be identified to the precision of 0.1. Although this is not a direct realization of the original Pólya-Hilbert conjecture, it provides an indirect observation of the Riemann zeros in a quantum system. In the future, this method can be extended to identify more zeros by improving the robustness and detection efficiency of the platform. Another promising route is to use a more efficient driving function for which the quasienergies decay slowly, for example, the Riemann function in the form used in Refs. [6,8].

\section{ACKNOWLEDGMENTS}

This work was supported by the National Key Research and Development Program of China (Grants No. 2017YFA0304100 and No. 2016YFA0302700), the National Natural Science Foundation of China (Grants No.11874343, 
No. 61327901, No. 11774335, No. 11474270, No. 11734015, and No. 11821404), Key Research Program of Frontier Sciences, CAS (Grant No. QYZDY-SSW-SLH003), the Fundamental Research Funds for the Central Universities (Grants No. WK2470000026, No. WK2470000027, and No. WK2470000028), and Anhui Initiative in Quantum Infor- mation Technologies (Grants No. AHY020100 and No. AHY070000). G.S. was supported by Grants No. PGC2018095862-B-C21, No. QUITEMAD+ S2013/ICE-2801, and No. SEV-2016-0597 (Spain) and the CSIC Platform on Quantum Technologies PTI-001. C.E.C. was supported by Spain's MINECO through Grant No. FIS2017-84368-P.
[1] B. Riemann, Über die Anzahl der Primzahlen unter einer gegebenen Grösse, Monatsberichte d. Preuss. Akad. d. Wissens., Berlin (1959), http://www.maths.tcd.ie/pub/HistMath/ People/Riemann/Zeta.

[2] C. L. Siegel, Über Riemanns nachlaß zur analytischen zahlentheorie, Quellen Studien zur Geschichte Math. Astron. Phys. Abt. B, Studien 2, 45 (1932) [reprinted in Gesammelte Abhandlungen (Springer, Berlin, 1966), Vol. 1].

[3] H. L. Montgomery, The pair correlation of zeros of the zeta function, Proc. Symp. Pure Math 24, 181 (1973).

[4] G. H. Hardy and J. E. Littlewood, Contributions to the theory of the Riemann zeta-function and the theory of the distribution of primes, Acta Math. 41, 119 (1916).

[5] X. Gourdon, The $10^{13}$ first zeros of the Riemann zeta function, and zeros computation at very large height (unpublished).

[6] B. van der Pol, An electro-mechanical investigation of the Riemann zeta function in the critical strip, Bull. Am. Math. Soc. 53, 976 (1947).

[7] M. V. Berry, Riemann zeros in radiation patterns: II. Fourier transforms of zeta, J. Phys. A: Math. Theor. 48, 385203 (2015).

[8] M. V. Berry, Riemann zeros in radiation patterns, J. Phys. A: Math. Theor. 45, 302001 (2012).

[9] C. Feiler and W. P. Schleich, Entanglement and analytical continuation: An intimate relation told by the Riemann zeta function, New J. Phys. 15, 063009 (2013).

[10] G. Pólya, Bemerkung über die integraldarstellung der Riemannschen $\zeta$-funktion, Acta Math. 48, 305 (1926).

[11] H. M. Edwards, Riemann's Zeta Function (Academic, New York, 1974), Vol. 58.

[12] M. L. Mehta, Random Matrices (Elsevier, Amsterdam, 2004), Vol. 142.

[13] F. W. Firk and S. J. Miller, Nuclei, primes and the random matrix connection, Symmetry 1, 64 (2009).

[14] A. Crisanti, G. Paladin, and A. Vulpiani, Products of Random Matrices: in Statistical Physics (Springer Science + Business Media, New York, 2012), Vol. 104.

[15] M. V. Berry and J. P. Keating, A rule for quantizing chaos, J. Phys. A: Mat. Gen. 23, 4839 (1990).

[16] E. Bogomolny, Riemann zeta function and quantum chaos, Prog. Theor. Phys. Suppl. 166, 19 (2007).

[17] M. V. Berry and J. P. Keating, The Riemann zeros and eigenvalue asymptotics, SIAM Rev. 41, 236 (1999).

[18] J. Kuipers, Q. Hummel, and K. Richter, Quantum Graphs Whose Spectra Mimic the Zeros of the Riemann Zeta Function, Phys. Rev. Lett. 112, 070406 (2014).

[19] D. Schumayer, B. P. van Zyl, and D. A. Hutchinson, Quantum mechanical potentials related to the prime numbers and Riemann zeros, Phys. Rev. E 78, 056215 (2008).
[20] M. Berry and J. Keating, A compact Hamiltonian with the same asymptotic mean spectral density as the Riemann zeros, J. Phys. A: Math. Theor. 44, 285203 (2011).

[21] D. Schumayer and D. A. Hutchinson, Colloquium: Physics of the Riemann hypothesis, Rev. Mod. Phys. 83, 307 (2011).

[22] B. T. Torosov, G. Della Valle, and S. Longhi, Quantum simulation of the Riemann-Hurwitz $\zeta$ function, Phys. Rev. A 87, 032103 (2013).

[23] G. Sierra and J. Rodríguez-Laguna, $H=x p$ Model Revisited and the Riemann Zeros, Phys. Rev. Lett. 106, 200201 (2011).

[24] G. Sierra, The Riemann zeros as spectrum and the Riemann hypothesis, Symmetry 11, 494 (2019).

[25] C. M. Bender, D. C. Brody, and M. P. Müller, Hamiltonian for the Zeros of the Riemann Zeta Function, Phys. Rev. Lett. 118, 130201 (2017).

[26] C. E. Creffield and G. Sierra, Finding zeros of the Riemann zeta function by periodic driving of cold atoms, Phys. Rev. A 91, 063608 (2015).

[27] N. H. Lindner, G. Refael, and V. Galitski, Floquet topological insulator in semiconductor quantum wells, Nat. Phys. 7, 490 (2011).

[28] A. G. Grushin, Á. Gómez-León, and T. Neupert, Floquet fractional Chern insulators, Phys. Rev. Lett. 112, 156801 (2014).

[29] X.-Y. Xu, Q.-Q. Wang, M. Heyl, J. C. Budich, W.-W. Pan, Z. Chen, M. Jan, K. Sun, J.-S. Xu, Y.-J. Han et al., Measuring a dynamical topological order parameter in quantum walks, Light Sci. Appl. 9, 7 (2020).

[30] K. Sacha, Modeling spontaneous breaking of time-translation symmetry, Phys. Rev. A 91, 033617 (2015).

[31] J. Zhang, P. Hess, A. Kyprianidis, P. Becker, A. Lee, J. Smith, G. Pagano, I.-D. Potirniche, A. C. Potter, A. Vishwanath et al., Observation of a discrete time crystal, Nature (London) 543, 217 (2017).

[32] G. Floquet, Sur les equations differentielles lineaires, Ann. Sci. Ecole Norm. Super. Suppl. 2 12, 47 (1883).

[33] J. H. Shirley, Solution of the Schrödinger equation with a Hamiltonian periodic in time, Phys. Rev. 138, B979 (1965).

[34] M. Grifoni and P. Hänggi, Driven quantum tunneling, Phys. Rep. 304, 229 (1998).

[35] A. Eckardt, M. Holthaus, H. Lignier, A. Zenesini, D. Ciampini, O. Morsch, and E. Arimondo, Exploring dynamic localization with a Bose-Einstein condensate, Phys. Rev. A 79, 013611 (2009).

[36] J. Johansson, P. Nation, and F. Nori, QuTiP: An open-source Python framework for the dynamics of open quantum systems, Comput. Phys. Commun. 183, 1760 (2012). 
[37] C. Creffield, Location of crossings in the Floquet spectrum of a driven two-level system, Phys. Rev. B 67, 165301 (2003).

[38] F. Grossmann, T. Dittrich, P. Jung, and P. Hänggi, Coherent Destruction of Tunneling, Phys. Rev. Lett. 67, 516 (1991).

[39] J.-M. Cui, Y.-F. Huang, Z. Wang, D.-Y. Cao, J. Wang, W.-M. Lv, L. Luo, A. Del Campo, Y.-J. Han, C.-F. Li et al., Experimental trapped-ion quantum simulation of the Kibble-Zurek dynamics in momentum space, Sci. Rep. 6, 33381 (2016).

[40] Y. Wang, M. Um, J. Zhang, S. An, M. Lyu, J.-N. Zhang, L.-M. Duan, D. Yum, and K. Kim, Single-qubit quantum memory exceeding ten-minute coherence time, Nat. Photon. 11, 646 (2017).
[41] L. E. de Clercq, R. Oswald, C. Flühmann, B. Keitch, D. Kienzler, H.-Y. Lo, M. Marinelli, D. Nadlinger, V. Negnevitsky, and J. P. Home, Estimation of a general time-dependent Hamiltonian for a single qubit, Nat. Commun. 7, 11218 (2016).

[42] J. von Neumann and E. Wigner, The Collected Works of Eugene Paul Wigner (Springer, Berlin, 1993), pp. 294-297.

[43] G. Della Valle, M. Ornigotti, E. Cianci, V. Foglietti, P. Laporta, and S. Longhi, Visualization of Coherent Destruction of Tunneling in an Optical Double Well System, Phys. Rev. Lett. 98, 263601 (2007).

[44] H. Lignier, C. Sias, D. Ciampini, Y. Singh, A. Zenesini, O. Morsch, and E. Arimondo, Dynamical Control of Matter-Wave Tunneling in Periodic Potentials, Phys. Rev. Lett. 99, 220403 (2007). 